\title{
Robustness of one-dimensional photonic band gaps under random variations of geometrical parameters
}

\author{
H. Sami Sözüer* and Koray Sevim \\ Izmir Institute of Technology, Department of Physics, Gulbahce Koyu, Urla, Izmir, Turkey \\ (Received 8 April 2005; revised manuscript received 8 August 2005; published 2 November 2005)
}

\begin{abstract}
The supercell method is used to study the variation of the photonic bandgaps in one-dimensional photonic crystals under random perturbations to thicknesses of the layers. The results of both plane wave and analytical band structure and density of states calculations are presented along with the transmission coefficient as the level of randomness and the supercell size is increased. It is found that with the supercell size fixed at 1024 unit cells, higher bandgaps disappear first as the randomness is gradually increased. The lowest bandgap is found to persist up to a randomness level of 55\%. However, as the supercell size is increased all bandgaps are observed to approach pseudogaps but with very low density of states. It is shown that harmonics of a relatively small cluster of closely spaced defects largely account for the bulk of the modes that populate the photonic bandgaps.

DOI: 10.1103/PhysRevB.72.195101

PACS number(s): 42.70.Qs, 41.20.Jb
\end{abstract}

\section{INTRODUCTION}

Since the pioneering work of Yablonovitch ${ }^{1}$ and John, ${ }^{2}$ research on photonic crystals (PCs) has enjoyed a nearly exponential increase. The manufacture of PCs at the optical regime has become a reality. ${ }^{3}$ Manufacturing brings with it the practical reality of random errors introduced during the manufacturing process and it is the effect of these random errors on the desirable features of PCs, namely photonic band gaps, that we wish to address in this paper.

Bandgaps in PCs depend on two crucial properties: an infinite and perfect translational symmetry. Clearly, in real life no crystal is infinite in size or perfectly periodic. When randomness is introduced in the geometry of the PC, one quantity of interest is the size of the bandgaps as the level of randomness is increased, and whether the bandgaps of the bulk perfect PC will survive the randomness. Same considerations apply for a finite PC. In fact, even for a perfect but finite PC, one needs to give up the notion of a bandgap and has to be content with severe depressions in transmittance instead. In this paper, we will consider both finite imperfect PCs by examining the dependence of their transmittance on randomness, and bulk imperfect PCs by determining their density of states (DOS) under varying degrees of randomness, using the supercell method. In particular, we will demonstrate the dramatic dependence of the size of the supercell on the DOS.

Although much has been done ${ }^{4-10}$ regarding imperfect two- and three-dimensional PCs, we feel that a study of the problem for one-dimensional (1D) PCs is warranted because of the inherent simplicity of the geometry and because of the availability of a variety of extremely accurate mathematical tools which allow a detailed study of the problem without having to compromise accuracy. For instance, because the electric field and its first derivative are continuous across the interface, and because of the low dimensionality of the 1D PC, the convergence problem that plagued band structure calculations for many 3D PCs (Refs. 11,12) is essentially nonexistent for 1D structures. Thus, we were able to use the old trusted plane wave (PW) method to find the band struc- ture and the DOS for supercell sizes not even imaginable in three- or even two-dimensional supercell calculations, ${ }^{4,6,7}$ One can obtain better than $0.1 \%$ convergence with as few as $\sim 30$ plane waves per unit cell in the supercell. The transmission coefficient can also be calculated for nearly arbitrary supercell sizes. Finally, one can calculate the band structure and the imaginary part of the wave vector using a semianalytical approach for very large supercells.

The 1D PC is, in many ways, the "infinite square-well" problem of photonic crystals. It contains the essential features of its bigger cousins in two and three dimensions without the mathematical complexities and the accompanying numerical uncertainties ${ }^{11,12}$ that can sometimes overshadow the essentials. For example, with 3D face centered cubic structures, it becomes practically impossible, due to convergence problems, to increase the supercell size beyond $2 \times 2 \times 2$ (or at most $3 \times 3 \times 3$ ) conventional cubic unit cells which contain only 32 primitive cells per supercell (or 108), since typically at least $\sim 1000$ terms per primitive cell are necessary to ensure sufficient convergence for inverse opal structures. It is not obvious from the start whether a randomness analysis with such small supercell sizes would yield results that are physically meaningful. ${ }^{7}$ In $2 \mathrm{D}$ calculations of imperfect PCs, larger supercells with sizes up to $\sim 15 \times 15$ have been employed $^{6}$ thanks to lower computational requirements for 2D problems.

Artifacts due to the small supercell size are bound to be inextricably intertwined with the physically significant bulk features of the imperfect PC. We show in Sec. II B that a superlattice constant of order $A \sim 10 a$ can be misleading in predicting the effects of randomness on the bandgaps. At large supercell sizes, we observe very interesting phenomena that cannot be observed with small superlattice constants. It is important to realize that the supercell is merely a computational instrument: with the supercell method one still calculates the bands of an infinite perfect $\mathrm{PC}$. The randomness is only within the supercell, but on a global scale, it is still a perfectly periodic structure with the supercell repeating itself ad infinitum. Since this infinite superperiodic structure is certainly not the physical structure that one is interested in, one 


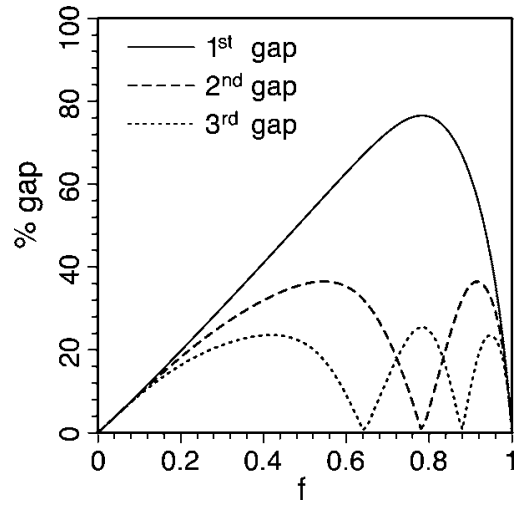

FIG. 1. The relative gap width vs the filling ratio $f$ for a 1D PC made of slabs of alternating dielectric constant of $\epsilon_{1}=1$ and $\epsilon_{2}$ $=13$. The lowest gap has a maximum for $f=1-\sqrt{\epsilon_{1} / \epsilon_{2}}=0.72$, which is the quarter-wave stack value. For this value of $f$, the even numbered gaps, the second, fourth, etc., which are in general nonzero for an arbitrary value of $f$ are all closed.

needs to be careful in distinguishing the artifacts of the superperiodic structure from the physical properties of the structure that one models within the supercell. To resolve the supercell artifacts from the physical features brought about by randomness, one needs to ensure that the interaction between neighboring supercells, which, to a good approximation, is proportional to the surface area of the supercell, be small compared to the bulk properties of the imperfect PC, which can be taken to be proportional to the volume of the supercell. Hence, on purely dimensional grounds, one can argue that the surface to volume ratio of the supercell $1 / A$, where $A$ is the superlattice constant, should be small compared to the typical length scale of the problem, namely, the inverse wavelength at the center of the gap. We allowed the supercell size $N$ to vary from $N=2$ to $N \approx 9000$, and one can clearly see the supercell artifacts gradually diminishing while the bulk features become more prominent in the limit as $N \rightarrow \infty$. On the other hand, 1D structures can have features, such as a bandgap for any geometry and any refractive index contrast, that are certainly not shared by $2 \mathrm{D}$ or $3 \mathrm{D}$ PCs, so one needs to be cautious in extrapolating the $1 \mathrm{D}$ results to $2 \mathrm{D}$ and $3 \mathrm{D}$ systems.

\section{THE IMPERFECT PC}

The precise distribution of randomness in the geometry of a PC would surely depend on the details of the specific manufacturing process. In the interest of simplicity, we chose the simplest distribution, the uniform distribution, in our study. As the unit cell, we chose a unit "supercell" that consisted of up to $\sim 16000$ unit cells. The thicknesses of the layers were perturbed by a given percent, by adding random numbers chosen from a uniform distribution. As the unperturbed structure, we chose the quarter-wave stack that has, for a given dielectric contrast, the largest relative gap between the first and the second bands, as can be seen in Fig. 1. In what follows, we will consider this structure with a dielectric contrast of 13 as our perfect PC. For 1D PCs, one further has the luxury of calculating the bandgaps using an analyti- cal method. ${ }^{13}$ This approach also permits the calculation of the imaginary part of the wave vector in the forbidden gap region and allows a reliable assessment of the accuracy of the plane wave method for the problem at hand.

We also investigated the transmission coefficient for a 250 unit cell quarter-wave stack structure. The transmission coefficient was calculated by simply matching the boundary conditions for the electric and the magnetic fields at each interface between the slabs in the multilayer structure.

\section{A. Density of states calculation with the PW method}

Maxwell's equations for waves propagating in the $x$ direction in a medium with a dielectric constant $\epsilon(x)$ that depends only on $x$, can be reduced to

$$
\frac{\partial^{2} E}{\partial x^{2}}-\frac{1}{c^{2}} \epsilon(x) \frac{\partial^{2} E}{\partial t^{2}}=0,
$$

where $E$ is parallel to the slabs. With $\epsilon(x)$ periodic along $x$ with lattice constant $a$, and translationally invariant along $y$ and $z$

$$
\epsilon(x)=\sum_{g} \epsilon(g) e^{i g x} \quad \text { with } \quad \epsilon(g)=\frac{1}{a} \int_{0}^{a} \epsilon(x) e^{-i g x} d x,
$$

where $g=m 2 \pi / a$ is a reciprocal lattice vector with $m=0$, $\mp 1, \mp 2, \ldots$, and $E(x)$ can be written as

$$
E(x)=e^{i k x} \sum_{g} E(g) e^{i g x},
$$

where $-\pi / a<k<\pi / a$. For a given $k$, this yields an $\infty$-dimensional generalized eigenproblem

$$
Q^{2} E=\frac{\omega^{2}}{c^{2}} \epsilon E
$$

or by multiplying both sides from the left by $Q \epsilon^{-1}$, one obtains the ordinary eigenproblem

$$
\left(Q \epsilon^{-1} Q\right)(Q E)=\frac{\omega^{2}}{c^{2}}(Q E),
$$

where $Q \equiv(k+g) \delta_{g g^{\prime}}, \epsilon_{g g^{\prime}} \equiv \epsilon\left(g-g^{\prime}\right)$, and $\epsilon^{-1}$ is the inverse of the matrix $\epsilon$. For a given value of $k$, a truncation of this $\infty$-dimensional ordinary, eigenvalue problem yields, by retaining only the $g$ vectors with $|g|<g_{\text {max }}$, the band structure $\omega_{j}(k)$ and the modes $E_{j k}(g)$. We choose a structure where the dielectric constant alternates between two values $\epsilon_{1}$ and $\epsilon_{2}$ each with thickness $d_{1}$ and $d_{2}$, respectively.

The choice of the lattice constant $a=d_{1}+d_{2}$ is not unique. Although the choice $a=d_{1}+d_{2}$ is the most obvious and the most convenient, the lattice constant can be chosen as any integer multiple of $d_{1}+d_{2}, A \equiv N a$. With a choice for $A$ with $N>1$, and following the same formalism one can write

$$
\epsilon(x)=\sum_{G} \epsilon(G) e^{i G x}
$$

with 




FIG. 2. The band structure of a perfect 1D PC with different choices of supercell size $N$. The parameters of the structure are those of a quarter-wave stack $\epsilon_{1}=13, \epsilon_{2}=1$, and $d_{1} / d_{2}=\sqrt{\epsilon_{2} / \epsilon_{1}}$. The points $\Gamma$ and $M$ of the "Brillouine zone" correspond to $K=0$ and $K=\pi / A$, respectively. When randomness is introduced, small gaps appear between each and every fold.

$$
\epsilon(G)=\frac{1}{A} \int_{0}^{A} \epsilon(x) e^{-i G x} d x
$$

and

$$
E(x)=e^{i K x} \sum_{G} E(x) e^{i G x},
$$

where $G=m(2 \pi / A)$, with $m=0, \mp 1, \mp 2, \ldots$, and $-\pi / A<K$ $<\pi / A$. Clearly, to get results with the same level of accuracy as before, i.e., with $N=1$, one would now need to include $N$ times as many plane waves in the expansion, which simply increases the computational burden, both in terms of storage and computing time. The band structure for $N=1,2$, 3 and 250 are displayed in Fig. 2 for a perfect PC. The folding of the bands in the first Brillouin zone for each $N$, makes the appearance of the bands rather different for each case, although the DOS and the eigenfunctions $E$ would be independent of the choice of the supercell size. The frequency is plotted in units of $2 \pi / a$ for all cases, so the frequency scale is not affected with the result that the bandgaps are at the same frequency, as would be expected.

To calculate the DOS, we choose a uniform mesh in $k$-space to calculate the bands and then choose a small frequency window $\Delta \omega$ and count the number of modes whose frequencies fall within that window. We add random perturbations to the thicknesses of the layers in the supercell such that

$$
d_{1,2}=d_{1,2}^{0}\left[1+2 p\left(u-\frac{1}{2}\right)\right],
$$

where $d_{1,2}^{0}$ are the unperturbed values of the thicknesses of the layers, i.e., the quarter-wave stack values, $u$ is a uniformly distributed random number in the interval $(0,1)$. We control the amount of disorder by varying the percent randomness parameter $p$ between 0 and 1. $p=0$ corresponds to perfectly periodic structures, and $p=1$ corresponds to a $100 \%$ fluctuation where $d_{1}, d_{2}$ can range between 0 and twice their unperturbed values. When disorder is introduced, gaps appear between every fold for $N>1$.

In Fig. 3 we plot the upper and lower limits for the lowest three bandgaps as a function of the percent randomness $p$, calculated with a supercell size of $N=1024$. Note that since for quarter-wave stack structures the even numbered gaps are closed, the bandgaps in this figure are in fact the first, third and the fifth bandgaps of a 1D PC with arbitrary values for the layer thicknesses. The third gap centered at $\omega a / 2 \pi c$ $=1.59$ closes around $p_{3}=0.1$, the second gap centered at $\omega a / 2 \pi c=0.96$ closes around $p_{2}=0.18$, and the lowest gap centered at $\omega a / 2 \pi c=0.32$ closes around $p_{1}=0.55$. The ratios of the critical values of randomness $p_{1}: p_{2}: p_{3}$ agree well with the ratios of the corresponding center gap frequencies $\omega_{3}: \omega_{2}: \omega_{1}$. This can be understood using the simple argument that when the random fluctuations in the thicknesses of the layers become comparable to the wavelength of the gap center, the bandgap disappears since the destructive interference responsible for the existence of the forbidden band depends on the long-range periodicity at that scale.

\section{B. Analytical method}

As discussed in detail in Ref. 13, for $n$ dielectric layers with thicknesses $d_{1}, \ldots, d_{n}$, with dielectric constants $\epsilon_{1}, \ldots, \epsilon_{n}$, for a given $\omega$, one can obtain the transfer matrix, defined by

$$
\left[\begin{array}{l}
E_{0} \\
E_{1}
\end{array}\right]=\left[\begin{array}{ll}
M_{11} & M_{12} \\
M_{21} & M_{22}
\end{array}\right]\left[\begin{array}{c}
E_{2 n} \\
E_{2 n+1}
\end{array}\right],
$$

where

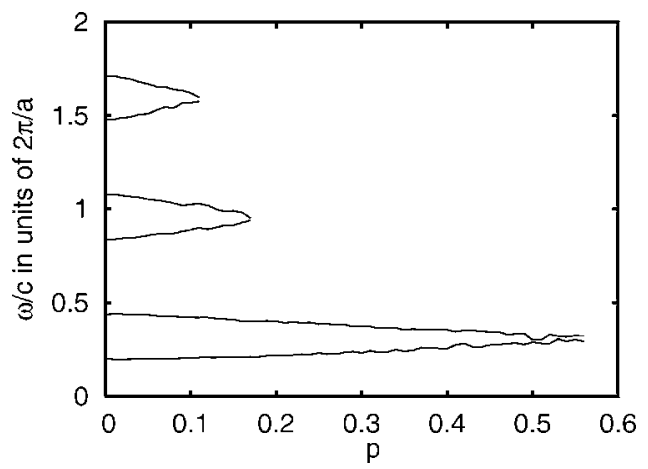

FIG. 3. The upper and lower band edges for the lowest three gaps calculated with a supercell of size $N=1024$, as a function of the disorder parameter $p$. 

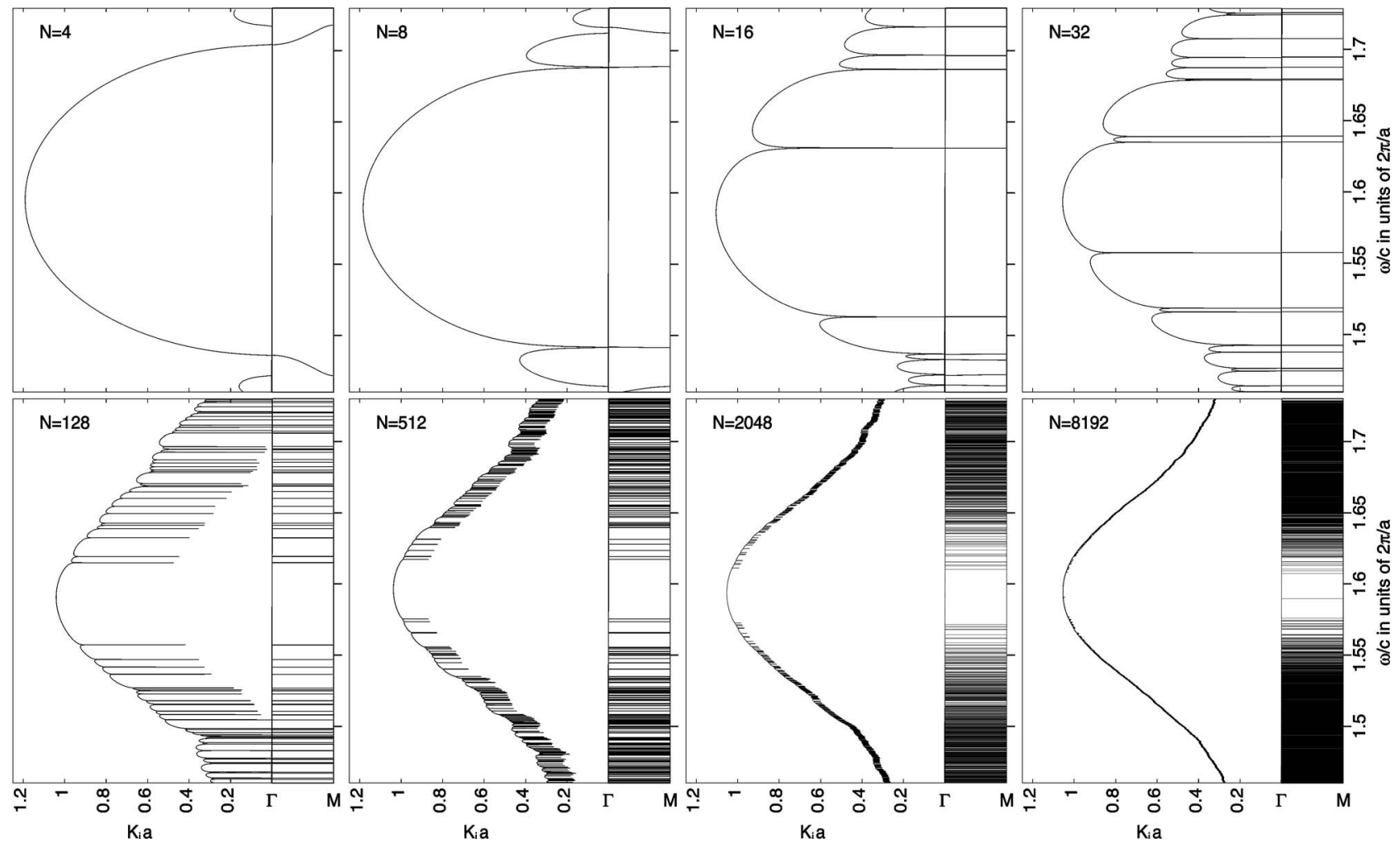

FIG. 4. The imaginary part of the wave vector $K_{i}$ and the band structure for selected values of the supercell size $N$ for a randomness level of $10 \%$. The band structure was calculated using the PW method and $K_{i}$ was calculated with the analytical method. Note the slight shift of the bands due to PW convergence. As $N$ grows, the third bandgap that lies between $1.474<\omega a / 2 \pi c<1.719$ in the perfect PC is more and more populated with transmission resonances, thereby narrowing the gap. For very large values of $N$, the bandgap appears to settle down to $1.58 \lesssim \omega a / 2 \pi c \lesssim 1.61$, although there is good indication that it would close at larger supercell sizes. For $N=2048$ and $N=8192$, the bands are not calculated as the PW method isn't practical for such large supercells. Instead, horizontal lines are drawn at the locations of the cusps of the $K_{i}$ vs $\omega$ plot to guide the eye. Note that the propagating bands rapidly approach measure zero as $N$ is increased (Ref. 5).

$$
M=D_{0}^{-1}\left(\prod_{l=1}^{n} D_{l} P_{l} D_{l}^{-1}\right) D_{n+1}
$$

with

$$
D_{l}=\left[\begin{array}{cc}
1 & 1 \\
\sqrt{\epsilon_{l}} & -\sqrt{\epsilon_{l}}
\end{array}\right] \text { and } P_{l}=\left[\begin{array}{cc}
e^{i \sqrt{\epsilon_{l}} \omega d_{l} / c} & 0 \\
0 & e^{-i \sqrt{\epsilon_{l}} \omega d_{l} / c}
\end{array}\right] .
$$

Imposing the Bloch condition on the $E$ field, one obtains

$$
\left[\begin{array}{c}
E_{0} \\
E_{1}
\end{array}\right]=e^{i K A}\left[\begin{array}{c}
E_{2 n} \\
E_{2 n+1}
\end{array}\right]
$$

Comparing with Eq. (6), the eigenvalues of the transfer matrix are seen to be $e^{i K A}$. Then, for $t \equiv\left|\left(M_{11}+M_{22}\right) / 2\right| \leqslant 1$, $K$ is real and is given by $K=(1 / A) \cos ^{-1} t$, while for $t>1, K$ is complex with $K_{r} A=\pi$ and $K_{i} A=-\ln \left(t-\sqrt{t^{2}-1}\right) .1 / K_{i}$ is the decay length of the evanescent mode, and is a measure of the strength of the bandgap. For finite PCs, it is desirable to have $K_{i} A \gg 1$ to have a significant drop in transmittance. The advantage of the exact method is that the supercell size $N$ can be increased to values that are practically impossible using the PW method. While with the PW method, using 30 plane waves per unit cell of the supercell, the memory requirements scale as $\sim(30 N)^{2}$, and the time requirements scale as $\sim(30 N)^{3}$, the exact method requires a very small amount of memory. The only disadvantage of the analytical method over the PW method is that, while in the PW method one chooses a real $K$ and calculates the frequencies corresponding to that value of $K$, in the analytical method, one chooses the frequency $\omega$ and calculates the real and imaginary parts of $K, K_{r}$, and $K_{i}$, corresponding to that value of $\omega$. If the bands are nearly flat, as is the case for very large supercell sizes, then one needs to sample the frequency interval of interest in very tiny increments in $\omega$ in order to "catch" a propagating mode. Thus the computation time can become very large. Also for large values of $N$, the transfer matrix $M$ can have very large elements so one requires very high precision in order to calculate the transmission resonance frequencies. We used quadruple precision (128-bit) floating point variables and functions in the Intel FORTRAN compiler in order to be able to resolve the transmission resonances for supercell sizes up to $N=8192$. For large values of $N$, even 128-bit precision is not sufficient, with the result that $K_{i}$ cannot be made to completely vanish due to insufficient precision. Nevertheless, the propagating modes appear as sharp cusps in the $K_{i}$ vs $\omega$ graph which can easily be identified (Fig. 4). For $N \gtrsim 8000$, one needs more than 128-bit precision to even see the cusps in Fig. 4. For larger values of $N$, we used MATHEMATICA for its arbitrary precision capabilities. 
However, compared to compiled code, MATHEMATICA is slower by several orders of magnitude, so we had to stop at around $N=32000$.

To understand the bulk features of imperfect PCs using the supercell method, one would need a large supercell, in fact the larger the better. As the supercell size is increased, small bandgaps begin to appear over regions that used to have propagating modes. One would normally expect the gaps of the perfect crystal to gradually shrink in size, rather than have more gaps, so this result seems somewhat puzzling at first sight. However, as the supercell size is increased, the statistical fluctuations decrease, and the pass bands become increasingly more densely populated.

In Fig. 4 we display the behavior of $K_{i}$ as $N$ is increased. As $N$ becomes larger what used to be a photonic bandgap becomes more and more populated with transmission resonances, and the forbidden gap edges gradually approach each other, narrowing the gap. It is possible that as $N \rightarrow \infty$, the whole bandgap region will be populated, albeit extremely sparsely, and instead of the bandgap, we will have a region where the DOS is extremely small-but nonzero nevertheless. We were able to increase up to $N=32768$ and the bandgap was reduced as $N$ became larger, although the decrease for very large $N$ values was very small. To actually see the gap narrow even more would require an impractically large $N$. It is quite obvious that a superlattice constant of $A \simeq 10 a$ is quite insufficient and yields an artificially large bandgap. One begins to get a more realistic picture for $A \gtrsim 100 a$.

The number of transmission branches in any finite frequency interval is proportional to $N$. The number of modes per branch can be calculated using

$$
n_{1}(\omega)=\int_{\text {branch }} \frac{d K}{d \omega} d \omega=\frac{\pi}{A}=\frac{\pi}{N a} .
$$

Thus the total number of propagating modes (per unit volume) is constant. As $N \rightarrow \infty$, these branches approach measure zero, and the group velocity approaches zero. It has been $\operatorname{argued}^{5}$ that these propagating modes cannot actually carry energy, and that the whole frequency region, in effect would be reflecting in the infinite imperfect PC limit.

Equally interesting is what happens to the propagating regions of the perfect PC. Small gaps appear in these regions and the propagating bands degenerate into many extremely narrow propagating branches. For finite but large PCs, because $K_{i}$ in the once-propagating regions is still much smaller than that in the gap regions as seen in Fig. 5, the long exponential tail of these mildly evanescent modes still tunnels through the finite PC and thus contributes to transmission in these regions. The passbands of the perfect PC too turn into small stopbands so the sharp distinction between the two types of bands is gradually blurred as randomness increases, and both types of bands become partly reflecting and partly transmitting. In the bandgap regions of the perfect $\mathrm{PC}$, the density of these transmission resonances is extremely small compared to that in the propagating regions, and these regions still appear to be more like bandgaps with a large, but still finite, supercell. Hence, it seems plausible to con- clude that one cannot speak of a "true bandgap" for imperfect PCs, but only of large depressions in the DOS, which, in practice, would serve the same purpose as bona fide bandgaps (see Fig. 6). For instance, for a cavity made of an "impurity" embedded in a PC, localized cavity modes would eventually leak out through the PC "walls" of finite thickness, regardless of how perfect the PC walls are, because of the finite thickness of the walls. For such an application, what is important is that the lifetime of the cavity mode be much larger than the relevant time scale. Since the lifetime of the cavity mode is a function of the transmittance, for a given value of transmittance, one would simply need to use thicker walls as the random perturbations are increased.

\section{Transmittance}

Practical applications must necessarily use finite sized PCs, and for such structures, a quantity of more relevance is the transmittance. We calculate the transmittance by considering a PC of $N$ unit cells, and each layer is perturbed as described earlier. The transmission coefficient is calculated by imposing the boundary conditions for $E D, B$, and $H$ at each layer boundary. This yields a set of $2 n+2$ linear equations for the unknowns $E_{1}, \ldots, E_{2 n+2}$. Setting the incident field $E_{0}=1$, and assuming vacuum dielectric values for the incident and transmitted fields, $\epsilon_{0}=\epsilon_{n+1}=1$, one obtains, $R$ $=E_{1}^{2}$ and $T=E_{2 n+2}^{2}$ for the reflection and transmission coefficients, respectively. Alternatively, one could also obtain $R$ and $T$ from the transfer matrix as detailed in Ref. 13 but with our approach, we can also obtain the $E_{i}$ within each layer. For a given frequency in the gap region, the dependence of $\ln T$ on the number of layers $N$ is approximately linear for all values of the randomness parameter $p$, as is the case ${ }^{13}$ for the perfectly periodic finite crystal (Fig. 7). However, depending on the level of randomness $\ln T$ can change by many orders of magnitude. In practice, this would mean that in order to obtain a given value of transmittance using an imperfect PC, one would now have to use a thicker PC.

Although there is a strong relationship between the transmittance of a finite imperfect PC, and the modes of the superlattice formed by choosing the same exact finite $\mathrm{PC}$ as the unit supercell, this relationship is not perfect in the sense that the existence of a propagating mode of the supercell does not necessarily imply a large value for $T$. A close examination of the $\ln T$ and $K_{i}$ vs $\omega$ plots in Fig 5 will reveal that, on a large scale, the transmission has a dip where $K_{i}$ is large, and when $K_{i}$ is small the transmittance is nearly unity. However, a closer look at a finer scale in Fig. 5, one sees that $\ln T$ can still be not as large as what one might expect from $K_{i}$. There is a subtle reason for this: The unit supercell can be chosen as any contiguous region of length $A$ of the underlying superperiodic structure, and the band structure is not affected by the choice of the supercell. However, for transmission calculations, one has to use a particular segment of length $A$ of this underlying superperiodic structure and depending on the choice, one would, in general, get a different transmittance for a given frequency. If a localized mode of the supercell is far from the incident side, then that mode may not show up on the transmittance spectrum. For instance, with a 

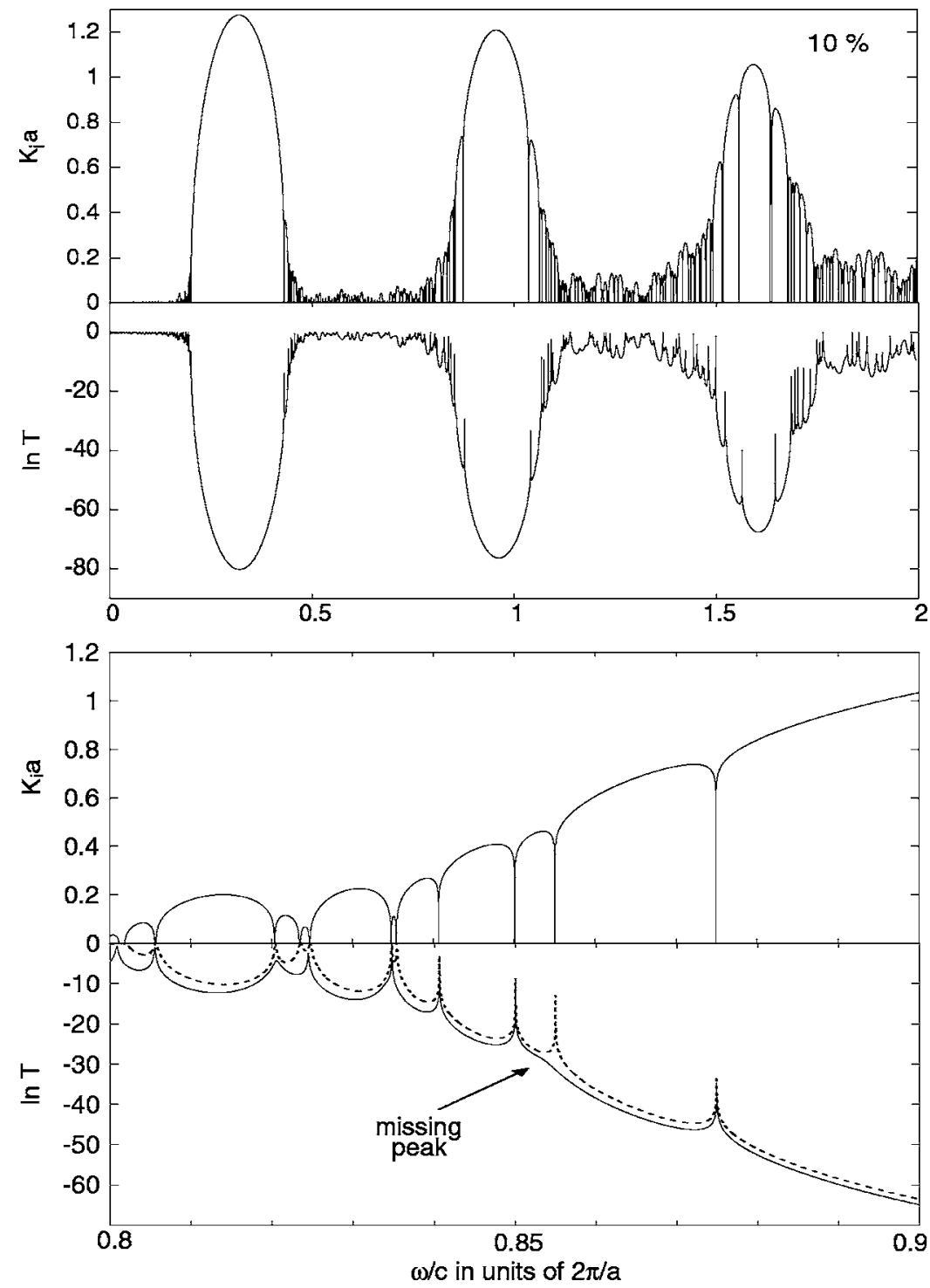

FIG. 5. (Left) $\ln T$ and $K_{i}$ vs $\omega$ for a supercell of size $N=32$ for a randomness level of $10 \%$. (Right) Closeup for $0.8<\omega a / 2 \pi c<0.9$ which contains the lower edge of the second gap at $0.83<\omega a / 2 \pi c<1.08$. The solid curve is for a particular ordering of the slabs of the supercell, while the dotted curve is the maximum value of $\ln T$ for all possible cyclic permutations of $\epsilon(x)$. The missing peak for the particular ordering is present in the supercell modes and in $\ln T_{\max }$.

single air slab defect centered at the supercell, one would observe both the transmission peak and the supercell mode. Shifting the supercell by $A / 2$ would split the defect at both ends of the supercell. While the supercell calculations are not affected by this cyclic permutation of the slabs, the transmission peak would disappear because the incident wave would see a perfectly periodic finite PC.

We shifted the supercell by small amounts for a given random structure and calculated the transmission spectrum for a single realization, as well as the maximum transmission, for each frequency, of all continuously cyclic permutations of $\epsilon(x)$. While the peaks of the maximum value of $\ln T$ agreed perfectly well with the supercell modes, some of the supercell modes did not appear in the transmission peaks of the single realization. This artifact of the supercell method clearly is not limited to 1D PCs and there can be significant differences between the supercell calculations and the trans- mission calculations for all PCs depending on just how the finite sample used in transmittance is cut out of the underlying superperiodic structure. A mode which is present for the bulk PC may not yield a high transmission value, because it would not couple to the mode due to incidence conditions. Similar coupling problems were reported by Robertson et al. ${ }^{14}$ for $2 \mathrm{D}$ PCs.

\section{DEFECT MODES}

In the imperfect PC model we studied, the thickness of each layer of the perfect PC was perturbed by a certain amount, in effect creating a defect at each lattice site. Thus, another way of attacking the imperfect PC problem is by starting from isolated single defects, and by gradually increasing the density of defects. Therefore it would make good sense to understand how a single defect affects the 


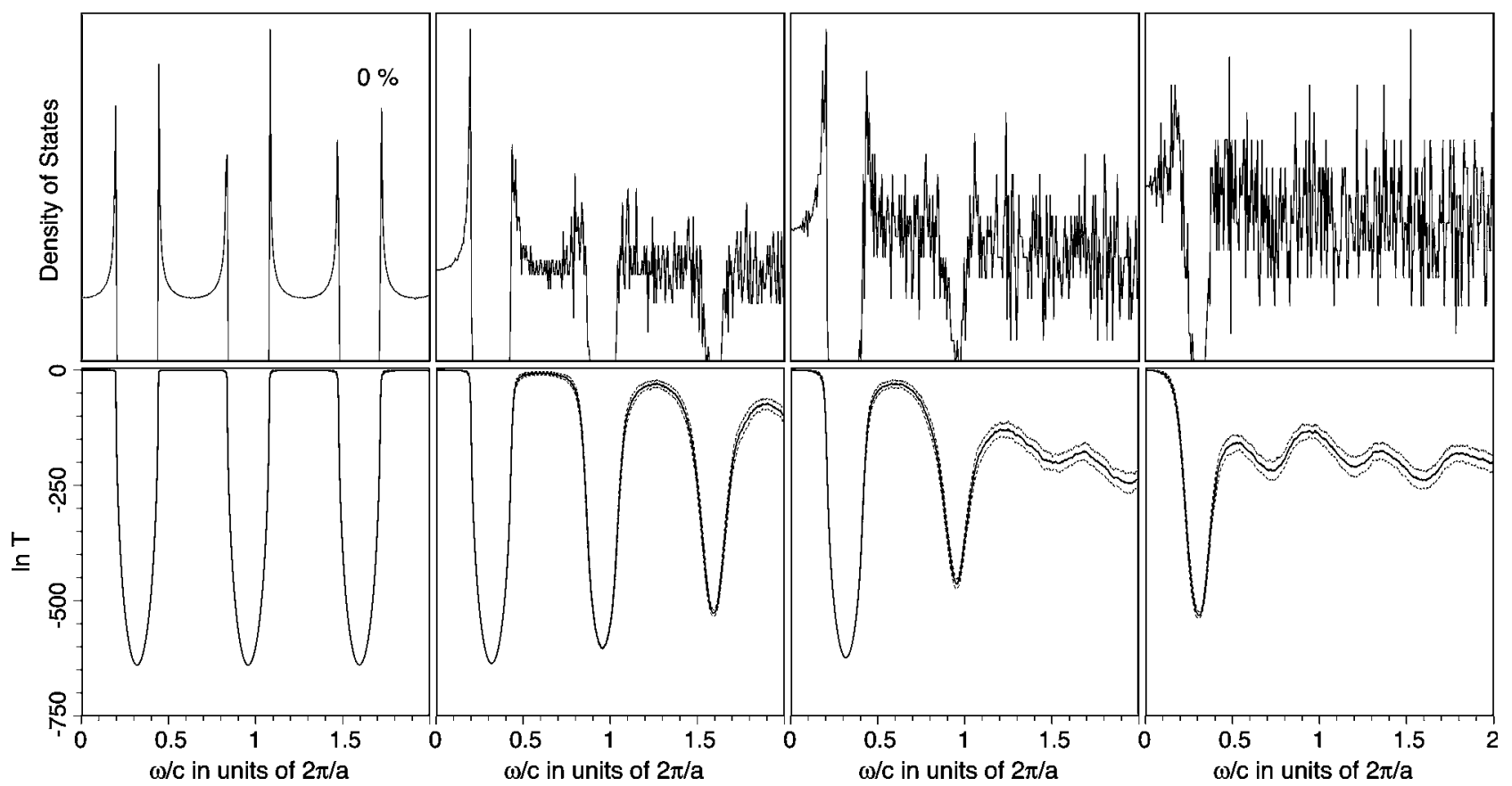

FIG. 6. The DOS and $\ln T$ vs frequency for various levels of randomness. $\ln T$ (solid curve) is the average for an ensemble of 100 random structures for each level of randomness. Also shown as dashed curves are $\ln T \pm \sigma$, where $\sigma$ is the standard deviation of $\ln T$ for the ensemble used.

band structure and the transmittance of an otherwise perfect PC. In Fig 8, we display the modes of a single defect of width $W=1.52 a$ sandwiched between 7 unit cells of perfect PC on either side. Each mode extends over a range of $\sim 9 a$. The first, third, and fifth harmonics of the defect modes fall nearly at the centers of the three gaps so these are the most sharply localized modes. When the mode frequency is near the gap edge, the modes are more widely distributed. One therefore would need a supercell of size of the order of $10 a$ to contain a single center-gap mode properly within the supercell. ${ }^{15}$ Increasing the supercell size further has essentially no effect on the single defect modes, except making the transmission peak sharper. Thus the supercell method works exceptionally well for a single defect, and the transmittance

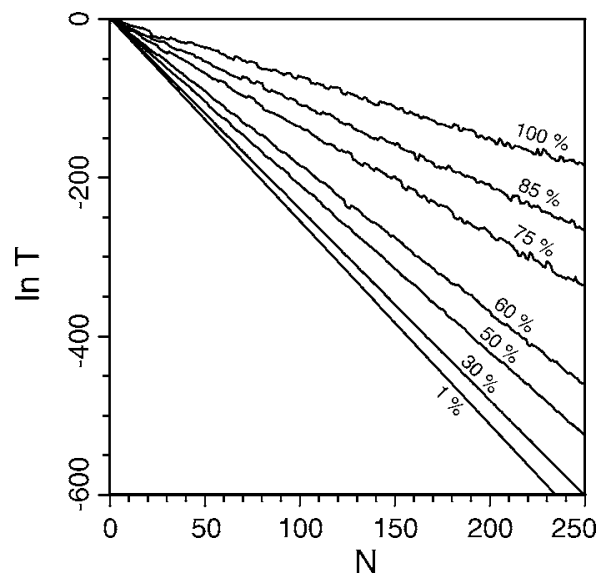

FIG. 7. The dependence of $\ln T$ on $N$ for different values of the randomness parameter $p$ for the center gap frequency of the first gap $w a / 2 \pi c=0.32$. and the supercell calculations agree well, provided the supercell chosen contains the defect modes reasonably well. When the defect modes from neighboring supercells overlap, one observes significant unphysical artifacts.

\section{A. Modes of a single defect}

We investigated the single-defect modes and their harmonics for various air slab defect sizes. Fig. 9(a) is a plot of the frequencies of the lowest harmonics as a function of $W / a$, where $a$ is the lattice constant and $W$ is the defect width. A simple clamped string of length $W$ would have modes such that $W=n \lambda / 2$, where $n=1,2, \ldots$, is the harmonic index. Then the $n$th harmonic frequency of the defect mode would be given by

$$
\left(\frac{\omega_{n} a}{2 \pi c}\right)=\frac{n}{2}\left(\frac{a}{W}\right), \quad n=1,2,3, \ldots
$$

We see from Fig. 9 that the defect mode frequencies agree perfectly with this simple model at the centers of the gaps, although there are significant deviations near the band edges. Figure 9(a) helps us understand, albeit qualitatively, the basic mechanism for the closing of the gaps. The value of $W / a$ $=[\sqrt{13} /(1+\sqrt{13})]=0.78287$ for which the structure is a perfect $\mathrm{PC}$ is marked with a vertical dashed line. For single isolated defects, the limits where $W$ deviates from the perfect PC value by $\pm 20, \pm 33.3$, and $\pm 100 \%$ are also marked as vertical dashed lines. At the $20 \%$ limit, the third harmonic and the second harmonic frequencies completely populate the third gap, the third harmonic frequencies moving in from above, and the second harmonic frequencies from below the center of the third gap, meeting at the gap center at $20 \%$ 


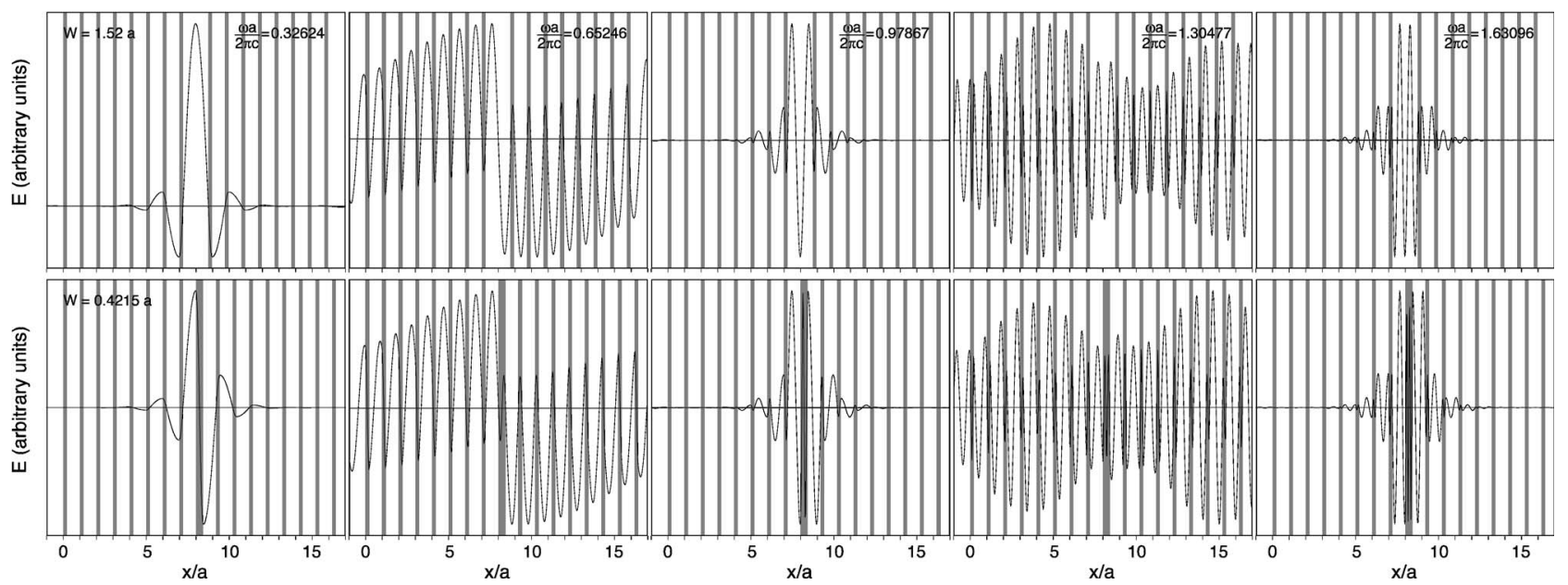

FIG. 8. The transmission $E$ fields for the lowest five defect mode harmonic frequencies for an air slab defect of width $W=1.52 a$ (upper row) sandwiched between eight unit cells of PC on each side, and for a dielectric slab defect of $W=1.52 a / \sqrt{13}=0.4215 a($ lower row). The gray bars indicate dielectric slabs with $\epsilon=13$. The parameters of the structure are those of a quarter-wave stack, $\epsilon_{1}=13, \epsilon_{2}=1$, and $d_{1} / d_{2}$ $=\sqrt{\epsilon_{2} / \epsilon_{1}}$. The two types of defects have the same optical path length and yield harmonics at exactly the same frequencies. The $E$ field of the air slab defect modes nearly vanish at the boundaries of the defect, thus resembling modes of a string clamped at both ends, while, for the dielectric slab defect modes, it is the slope of the $E$ field that nearly vanishes at the boundaries, similar to the modes of a string with both ends free. The modes for both types of defects, however, extend over several lattice constants at both sides. For the chosen defect width, the first, third, and the fifth harmonics fall nearly at the centers of the three bandgaps, and hence the corresponding modes are localized, while the second and the fourth harmonics are within pass bands, although the characteristic standing wave pattern inside the defect is still visible for these modes. The frequencies of the higher harmonics are nearly exact integer multiples of the fundamental mode, as expected.

randomness. Thus, had the defects been so sparsely located that the modes of individual defects had essentially no overlap, the third bandgap would have closed at a randomness level of $20 \%$. Similarly, the second gap would have closed at a randomness level of $33 \%$, with the second harmonic modes populating the upper half of the bandgap while the lowest

harmonic modes populate the lower half. The lowest gap would have closed at a randomness level of $100 \%$, with the first harmonic modes seeping into the gap from above. The modes that populate the first gap from below do not seem to be associated with any of the harmonics, as given by Eq. (9). These modes, whose frequencies are centered at the gaps for

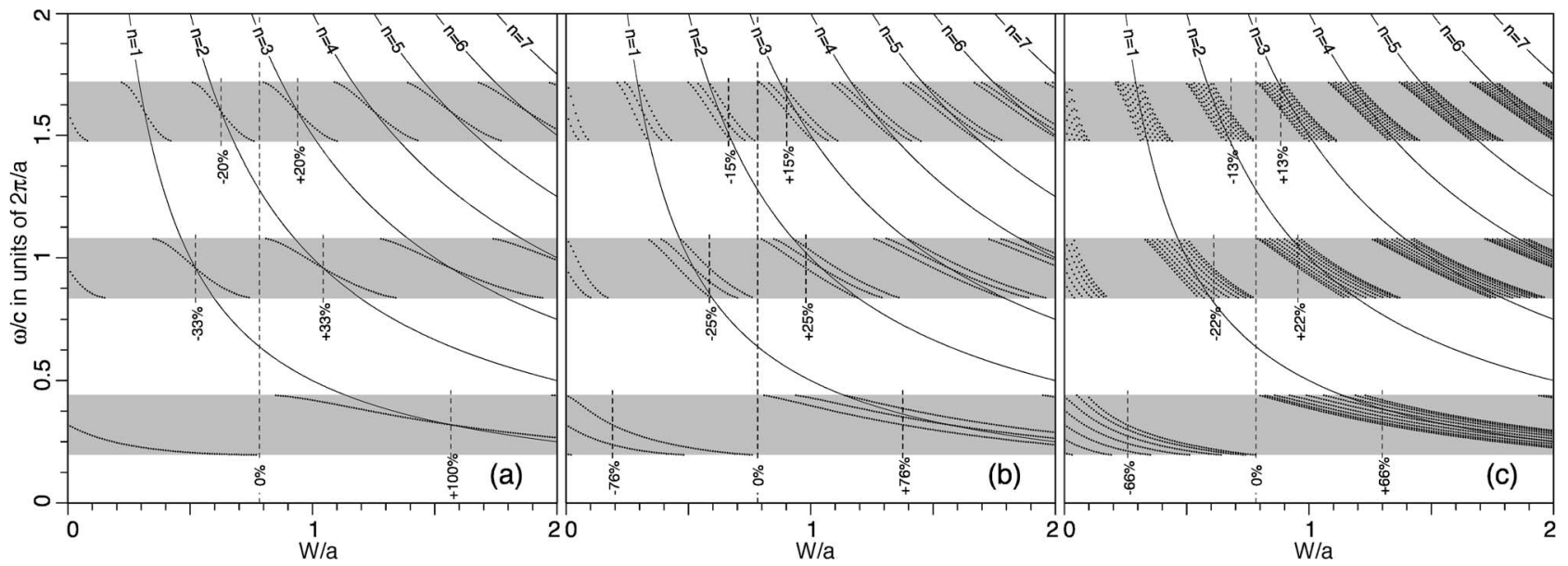

FIG. 9. The frequencies of the localized defect modes as a function of $W$, the defect width, for clusters of (a) one, (b) three, and (c) nine identical defects as calculated from the peaks of the transmission spectrum (dotted curves). The spacing between the air defects is only one dielectric slab for the defect clusters. The vertical dashed line is when the defect slab has a width that is equal to the perfect PC value. The vertical dashed lines show values of $W / a$ for which the deviation from the perfect PC value is the percentage indicated. The solid curves are the various harmonic frequencies of a $1 \mathrm{D}$ cavity given by Eq. (9), with $n=1$ corresponding to the fundamental frequency. The modes around $W / a=0$ do not belong to an air slab defect. They are the modes of a dielectric slab of twice the normal width when the air slab width vanishes and the two neighboring slabs fuse to form a single dielectric slab defect. The horizontal gray bars are the bandgap regions of the perfect PC. Notice how the individual defect modes are transformed into bands as the cluster size increases. Increasing the cluster size further has little effect on the widths of the bands and hence on the size of the bandgaps. Although these curves were calculated for air slab defects, one obtains the same curves also for dielectric slab defects, provided $W$ is replaced with the optical path length of the defect $W \sqrt{\epsilon_{d}}$. 

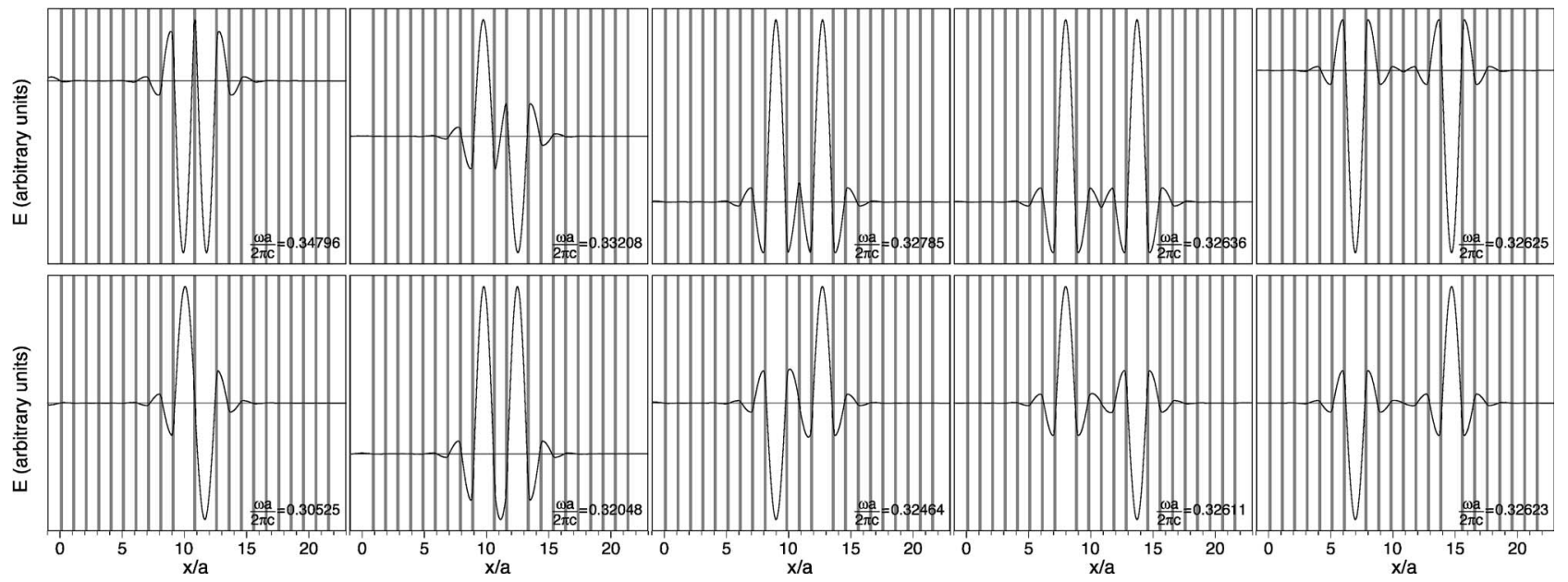

FIG. 10. The $E$ fields for the fundamental modes of two defects, each of width $W=1.52 a$, separated by various distances. The parameters of the structure are as in Fig. 7. Note the splitting of the single defect modes as the defect spacing is reduced.

$W=0$, are the modes of the defect one would have when the air defect vanishes and the two neighboring dielectric slabs fuse to form a single dielectric slab defect with a width that is twice the perfect PC value. We find that all three gaps close at much lower values of randomness than predicted by the single-defect model: 55,18 , and $11 \%$, respectively, for the first, second, and third gaps. So one would need to refine the model for a better understanding as to how the gaps close.

\section{B. Modes of a cluster of identical defects}

The disorder we study is evenly distributed over the entire crystal: the widths of each and every slab in the PC is randomly perturbed. Therefore it is not surprising that the single isolated defect model makes quantitatively inaccurate predictions. To refine the single-defect model, we considered a cluster of several equally spaced identical defects sandwiched between large enough perfect PC on both sides. We calculated the transmission spectra of these defect clusters as the interdefect spacing within the cluster was varied. Figure 10 is a plot of the transmission resonance modes for two identical defects with the interdefect spacing varied. The single defect modes, say the fundamental mode are degenerate when the spacing between neighboring defects is large enough so as to make the overlap of modes from neighboring defects insignificant. When the interdefect spacing is made smaller, however, the degeneracy is lifted and one observes the individual defect mode frequencies splitting into bands.

With a cluster of three defects separated by only one dielectric slab, each single-defect harmonic splits into three branches. With these additional modes, the bandgaps now close at 15,25 , and $76 \%$ for the third, second, and first bandgaps, respectively [Fig. 9(b)]. With nine defects the splitting of each harmonic is much more prominent as can be seen in Fig. 11. With an interdefect spacing of one dielectric slab from each other, we obtain the much better estimates 13 , 22 , and $66 \%$, for the critical values of randomness at which the third, second, and first gaps close, respectively [Fig. 9(c)]. Increasing the cluster size further to 27 and 81 had little effect on the critical randomness values. Although these figures are still not exact, it is remarkable that the simple deterministic model built from a cluster of $\sim 10$ closely spaced defects can account for the outstanding features of a complex stochastic problem. This can be exploited for investigating the effects of randomness in two- and threedimensional PCs, since the supercell method is much more suitable for finding the modes of a few defects. Notice that the critical values of randomness at which the gaps close have the ratio $1: 3: 5$ as expected from the ratio of the center gap frequencies for the three gaps.

\section{CONCLUSION}

We studied the behavior of the photonic bandgaps and the transmittance for an imperfect PC using the supercell method combined with both the plane wave method and the analyti-

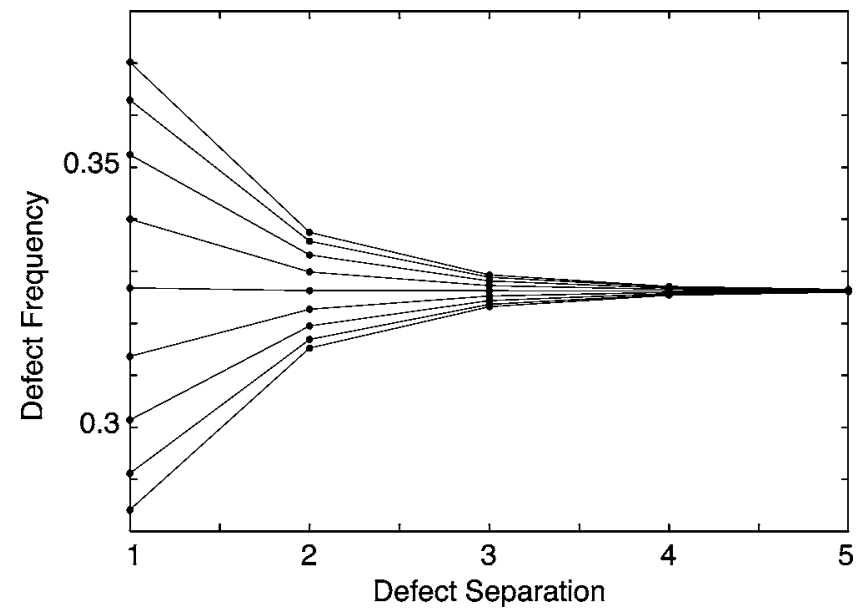

FIG. 11. Splitting of the lowest harmonic defect frequency for a cluster of nine equally spaced identical air slab defects, each of width $W=1.52 a$, sandwiched between large perfect PCs on either side. The abscissa is the number of dielectric slabs between the air defects. Note the wide splitting as the defect separation becomes smaller. 
cal method. Our results indicate that as randomness is increased, the distinction between the stopbands and the passbands of the perfect PC becomes increasingly blurred, with propagating modes appearing within the stopbands and the imaginary wave vector $K_{i}$ gradually decreasing. Conversely, the passbands of the perfect PC gradually turn into stop bands with the imaginary wave vector $K_{i}$ increasing and the density of propagating modes decreasing as randomness is increased. The whole frequency region tends to become reflecting at high values of randomness, with the propagating branches approaching measure zero. The bandgaps of the perfect PC are replaced by a DOS that is extremely small for small values of randomness. Unlike previously believed, the bandgaps do not narrow with increasing randomness, eventually closing at a certain randomness level. Rather, they turn into pseudogaps and the DOS gradually increases within the gaps while decreasing in the passband regions with increasing disorder. Using a small supercell, one gets artificially large bandgaps for a given randomness level and this can be misleading as to the size or the presence of bandgaps. The higher frequency bandgaps are populated first with the lowest gap closing at around a randomness level of $55 \%$.

We also investigated the modes of single defects and small clusters of defects and found that the closing of the bandgaps can be understood quite well by the harmonics of the modes of small, closely spaced defect clusters appearing within the gap regions of the perfect PC. This can be a crude but useful tool in analyzing disordered 2D and 3D PCs.

\section{ACKNOWLEDGMENTS}

This work was supported by a grant from the Research Fund at Izmir Institute of Technology. The band structure computations were performed on the 128-node Beowulf cluster at TUBITAK, the Scientific and Technological Research Council of Turkey.
*Electronic address: sozuer@photon.iyte.edu.tr

${ }^{1}$ E. Yablonovitch, Phys. Rev. Lett. 58, 2059 (1987).

${ }^{2}$ S. John, Phys. Rev. Lett. 58, 2486 (1987).

${ }^{3}$ S. Y. Lin, J. G. Fleming, D. L. Hetherington, B. K. Smith, R. Biswas, K. M. Ho, M. M. Sigalas, W. Zubrzycki, S. R. Kurtz, and J. Bur, Nature (London) 394, 251 (1998).

${ }^{4}$ Z. Y. Li and Z. Q. Zhang, Phys. Rev. B 62, 1516 (2000).

${ }^{5}$ A. P. Vinogradov and A. M. Merzlikin, Phys. Rev. E 70, 026610 (2004).

${ }^{6}$ E. Lidorikis, M. M. Sigalas, E. N. Economou, and C. M. Soukoulis, Phys. Rev. B 61, 13458 (2000); Y. S. Chan, C. T. Chan, and Z. Y. Liu, Phys. Rev. Lett. 80, 956 (1998).

${ }^{7}$ Z. Y. Li, X. Zhang, and Z. Q. Zhang, Phys. Rev. B 61, 15738 (2000)

${ }^{8}$ S. Fan, P. R. Villeneuve, and J. D. Joannopoulos, J. Appl. Phys. 78, 1415 (1995).
${ }^{9}$ M. M. Sigalas, C. M. Soukoulis, C. T. Chan, R. Biswas, and K. M. Ho, Phys. Rev. B 59, 12767 (1999).

${ }^{10}$ M. A. Kaliteevski, J. M. Martinez, D. Cassagne, and J. P. Albert, Phys. Rev. B 66, 113101 (2002); A. A. Asatryan, P. A. Robinson, L. C. Botten, R. C. McPhedran, N. A. Nicorovici, and C. MartijndeSterke, Phys. Rev. E 62, 5711 (2000).

${ }^{11}$ H. S. Sözüer, J. W. Haus, and R. Inguva, Phys. Rev. B 45, 13962 (1992).

${ }^{12}$ A. Moroz, Phys. Rev. B 66, 115109 (2002).

${ }^{13}$ Pochi Yeh, Optical Waves in Layered Media (Wiley, New York, 1988).

${ }^{14}$ W. M. Robertson, G. Arjavalingam, R. D. Meade, K. D. Brommer, A. M. Rappe, and J. D. Joannopoulos, Phys. Rev. Lett. 68, 2023 (1992).

${ }^{15}$ K. Sakoda and H. Shiroma, Phys. Rev. B 56, 4830 (1997). 